\title{
Homöopathie in der medizinischen Versorgung
}

\section{Präambel}

In den Therapie-Algorithmen unterschiedlichster Erkrankungen bildet sich zunehmend ein differenziertes pathophysiologisches Verständnis ab: Erkrankungen und Symptome sind vielfach nicht monokausal erklärbar, sondern ein Resultat von Fehlregulationen auf somatischer und psychischer Ebene. Das Verhalten von Patienten kann dabei sowohl Ursache als auch Folge der Krankheit sein. Zusätzlich ändern sich das Gesundheitsbewusstsein und das Krankheitsverständnis: Vielfach haben Patienten eine höhere Gesundheitskompetenz verbunden mit der Bereitschaft, mehr Eigenverantwortung zu übernehmen. Im Gegenzug fordern sie die Berücksichtigung ihrer individuellen Bedürfnisse, Präferenzen und Erfahrungen.

Das Konzept der integrativen Medizin wird diesem weiterentwickelten Krankheitsverständnis von Behandlern und Patienten gerecht, indem es leitlinienorientierte Ansätze und Behandlungskonzepte der Komplementärmedizin patientenzentriert anwendet. Wenn integrative Medizin neben der gesellschaftlichen Wertschätzung auch eine starke wissenschaftliche Akzeptanz erfahren soll, muss sie sich einer wissenschaftlichen Evaluation stellen. Das vorliegende Konsensus-Papier zeigt für die Homöopathie als Baustein eines integrativen Behandlungskonzeptes anhand vorliegender Daten ihre Möglichkeiten und Grenzen auf.

\section{Konsens}

1. Gesicherte Qualität. Homöopathische Medikamente sind standardisierte, qualitätsüberprüfte Arzneimittel. Ihre gleichbleibende pharmazeutische Qualität und Unbedenklichkeit wird durch das Arzneimittelrecht, die Herstellungsvorschriften und die Überwachungsbehörden gewährleistet. Die Anforderungen an Qualität und Sicherheit sind mit denen für chemisch-synthetische Arzneimittel vergleichbar [1].

2. Personalisierte Medizin. Die Homöopathie beachtet die individuelle Manifestation einer Erkrankung, indem nach dem Ähnlichkeitsprinzip ein passendes homöopathisches Arzneimittel gesucht wird, das die individuellen körperlichen und geistigen Symptome des Patienten abdeckt. Die methodische Anwendung dieses Prinzips zur Induktion von Heilungsprozessen macht die Homöopathie nachvollziehbar [2, 3].

3. Plausibles Modell zum Wirkprinzip. Die homöopathische Behandlung verfolgt das Ziel, die körpereigene Regulation und Selbstheilungskräfte anzuregen sowie physiologische Funktionen wiederherzustellen [2]. Es werden sowohl niedrige Potenzen mit höheren Konzentrationen als auch Hochpotenzen verwendet. Die Potenzstufe D8 entspricht rechnerisch etwa der Blutserumkonzentration des Schilddrüsenhormons FT3 oder des Parathormon [4, 5]. Zum Wirkmechanismus der höheren Potenzen gibt es vielversprechende Hypothesen [6-8], die jedoch noch bewiesen werden müssen, und es bedarf zu deren Verständnis eines modernen Substanzbegriffes, wie ihn die moderne Physik seit fast 100 Jahren entwickelt hat.

4. Wirksamkeit bei chronischen Erkrankungen. Bei chronischen Erkrankungen zeigen Kohortenstudien und Fallserien aus der Praxis eine gute und teilweise jahrelang nachhaltige Wirksamkeit, z. B. bei allergischer Rhinitis, Migräne, atopischer Dermatitis oder rezidivierenden Harnwegsinfekten [915]. Der Schweregrad wird signifikant reduziert, gleichzeitig erhöht sich die Lebensqualität [9-11].

5. Wirksamkeit bei akuten Erkrankungen. Bei bestimmten akuten Erkrankungen, z. B. oberen Atemwegsinfekten, kann die Homöopathie als Monotherapie wirksam eingesetzt werden [16]. Eine adjuvante Therapie kann die Schwere der Symptomatik reduzieren, die Krankheitsdauer signifikant verkürzen und chemisch-synthetische Arzneimittel einsparen [17-20].

6. Verbesserung der Lebensqualität. Placebo-kontrollierte klinische Prü- fungen weisen eine signifikante Verbesserung der Lebensqualität bei Mono- wie auch bei Kombinationstherapie mit homöopathischen Arzneimitteln nach [21, 22]. Additiv zur leitliniengerechten Behandlung eingesetzte homöopathische Arzneimittel können z. B. bei Tumorerkrankungen den Allgemeinzustand signifikant verbessern und Nebenwirkungen der konventionellen Therapie lindern [22-28].

7. Belegte Arzneimittelsicherheit. Unerwünschte Arzneimittelwirkungen sind meist leicht bis moderat und vorübergehend [29-31]. Damit ist die Homöopathie insbesondere auch für vulnerable Patienten/-innen wie Kinder, Ältere, Multimorbide und multimedikamentös Behandelte geeignet [9, 32-36].

8. Hohe Patientenzufriedenheit. Aufgrund positiver Erfahrungen wird die Homöopathie im Behandlungsalltag oft von Patienten/-innen nachgefragt [11, 37-39]. Es besteht eine hohe Akzeptanz und Zufriedenheit mit der homöopathischen Behandlung [40].

9. Qualifizierende Fort- und Weiterbildung unverzichtbar. Die homöopathische Behandlung ist individuell und bedarf einer fundierten Ausbildung. Ärzte/-innen absolvieren die ZusatzWeiterbildung Homöopathie, um ihre Patienten/-innen qualifiziert über Möglichkeiten und Grenzen der Homöopathie beraten zu können. Ärztliches Vertrauen in die Therapie und Interesse an dieser Weiterbildung sind hoch [38, 41].

10. Sozioökonomische Relevanz. Aktuelle Daten belegen, dass die homöopathische Behandlung in geeigneten Anwendungsbereichen gegenüber rein konventionellen Therapien bei guter Wirksamkeit häufig eine bessere Wirtschaftlichkeit zeigt. Die Ressourcen des Gesundheitswesens werden geschont und auch Patienten/-innen berichten über geringere Therapiekosten bei gleichzeitig höherer Lebensqualität [42-44]. 


\section{Resümee}

Qualität und Unbedenklichkeit homöopathischer Arzneimittel sind belegt und durch deren gesetzliche Verankerung als Arzneimittel gewährleistet. Homöopathie ist heute eine wissenschaftsfundierte, individualisierte Medizin. Klinische Studien zeigen, dass homöopathische Arzneimittel bei chronischen sowie akuten Indikationen eine wirksame und sozioökonomisch sinnvolle Alternative oder Ergänzung zu chemisch-synthetischen Arzneimitteln sein können. Vertrauen und Akzeptanz in die Homöopathie sind bei Ärzten/-innen wie auch Patienten/-innen hoch. Fort- und Weiterbildungskurse werden entsprechend häufig nachgefragt, um qualifiziert über die Möglichkeiten und Grenzen dieser Therapieform beraten zu können. Insgesamt wird deutlich, dass die Homöopathie im Sinne einer patientenzentrierten, pluralistischen Medizin und pragmatisch orientierten Therapiefreiheit einen wertvollen Beitrag zur Gesundheitsversorgung leisten kann.

\section{Interessenkonflikt}

Die Autorinnen/Autoren geben an, dass kein Interessenkonflikt besteht.

\section{Moderator}

\section{Prof. Dr. med. André-Michael Beer}

Direktor der Klinik für Naturheilkunde der Klinik Blankenstein, Hattingen

\section{Weitere Experten}

Prof. Dr. med. Dr. Ulrich Borchard Institut für Pharmakologie und Toxikologie Universität Düsseldorf

\section{Prof. Dr. med. Michael Frass}

Facharzt für Innere Medizin und internistische Intensivmedizin, Präsident des Österreichischen Dachverbandes für ärztliche Ganzheitsmedizin

\section{Prof. Dr. med. Ingrid Gerhard}

Fachärztin für Frauenheilkunde, Naturheilverfahren, Umweltmedizin, Heidelberg

\section{Prof. Dr. med. Roman Huber}

Universitätsklinikum Freiburg, Institut für Infektionsprävention und Krankenhaushygiene, Uni-Zentrum Naturheilkunde, Umweltmedizinische Ambulanz

\section{Prof. Dr. Michael Keusgen}

Pharmazeutische Chemie, Universität Marburg, Vorsitzender der HOM-Working Party des Europäischen Arzneibuches, Mitglied der Kommission D des Bundesinstituts für Arzneimittel und Medizinprodukte (BfArM)

\section{Prof. Dr. med. David Martin}

Facharzt für Kinder- und Jugendmedizin, Kinderendokrinologie \& -Diabetologie, Kinderonkologie \& -Hämatologie, Lehrstuhlinhaber, Gerhard-Kienle-Lehrstuhl (GKLS) für Medizintheorie, integrative und anthroposophische Medizin, Co-Leiter, Forschungs- und Lehrzentrum Herdecke (FLZ), Leiter, Institut für Integrative Medizin (IfIM), Universität Witten/Herdecke

\section{Prof. Dr. med. Harald Matthes}

Ärztlicher Leiter/Geschäftsführer am Gemeinschaftskrankenhaus Havelhöhe, Medizinische Klinik, akademisches Lehrkrankenhaus der Charité-Universitätsmedizin Berlin

\section{Prof. Dr. med. Jürgen Pannek}

Chefarzt Neuro-Urologie, Schweizer Paraplegiker-Zentrum Nottwil

Prof. Dr. Dr. med. Reinhard P. T. Rychlik Institut für Empirische Gesundheitsökonomie (IFEG), Medizinische Fakultät der Ruhr-Universität Bochum, Sachverständiger des Deutschen Bundestages und Gutachter der Europäischen Union im Bereich Public Health und Gesundheitsökonomie

\section{Literatur}

[1] Orth $\mathrm{H}$, Pannenbecker $\mathrm{A}$, Prato $\mathrm{N}$ et al. Pharmaceutical Quality of Homeopathic Medicinal Products. Pharm Ind 2021; 83 : 74-83

[2] Bullemer U, Beer A. Von der Materia medica bis zur Mittelwahl. Überprüfen Sie Ihr homöopathisches Wissen. MMW Fortschr Med 2009; 151: 38-40

[3] Teut M, Dahler J, Lucae C, Koch U. Kursbuch Homöopathie. 1. Auflage. München: Urban \& Fischer Verlag, Imprint der Elsevier $\mathrm{GmbH} ; 2008$.

[4] Neumeister B, Besenthal H, Liebich $\mathrm{H}$, Böhm BO. Klinikleitfaden Labordiagnostik. 3. Auflage ed. München: Urban \& Fischer Verlag, Imprint der Elsevier GmbH; 2003.

[5] Elsenhans I. Normalwerte in der Labordiagnostik. Aus Hahn JM. Checkliste Innere Medizin, Thieme Verlag 2006. Im Internet: https://m.thieme.de/viamedici/klinikfaecher-klinische-chemie-1536/a/klinischechemie-labor-normalwerte-3886.htm; Stand: 14.02.2021

[6] Klein SD, Würtenberger $S$, Wolf $U$ et al. Physicochemical Investigations of Homeo- pathic Preparations: A Systematic Review and Bibliometric Analysis-Part 1. J Altern Complement Med 2018; 24: 409-421

[7] Tournier A, Klein SD, Würtenberger $S$ et al. Physicochemical Investigations of Homeopathic Preparations: A Systematic Review and Bibliometric Analysis-Part 2. J Altern Complement Med 2019; 25: 890-901

[8] Tournier A, Würtenberger S, Klein SD et al. Physicochemical Investigations of Homeopathic Preparations: A Systematic Review and Bibliometric Analysis-Part 3. J Altern Complement Med 2021; 27: 45-57

[9] Witt CM, Lüdtke R, Baur R et al. Homeopathic medical practice: long-term results of a cohort study with 3981 patients. BMC Public Health 2005; 5: 115

[10] Witt CM, Lüdtke R, Mengler $N$ et al. How healthy are chronically ill patients after eight years of homeopathic treatment? - Results from a long term observational study. BMC Public Health 2008; 8: 413

[11] Spence DS, Thompson EA, Barron S]. Homeopathic treatment for chronic disease: a 6-year, university-hospital outpatient observational study. J Altern Complement Med 2005; 11: 793-798

[12] Pannek J, Pannek-Rademacher S, Jus MS et al. Usefulness of classical homeopathy for the prophylaxis of recurrent urinary tract infections in individuals with chronic neurogenic lower urinary tract dysfunction. J Spinal Cord Med 2019; 42: 453-459

[13] Gaertner K, von Ammon K, Frei-Erb M. Individualized Homeopathic Treatment in Women with Recurrent Cystitis: A Retrospective Case Series. Complement Med Res 2020; 27: 193-199

[14] Witt CM, Lüdtke R, Willich SN. Homeopathic treatment of children with atopic eczema: a prospective observational study with two years follow-up. Acta Derm Venereol 2009; 89: 182-183

[15] Witt CM, Lüdtke R, Willich SN. Homeopathic treatment of chronic headache (ICD-9: 784.0)--a prospective observational study with 2-year follow-up. Forsch Komplementmed 2009; 16: 227-235

[16] Haidvogl M, Riley DS, Heger M et al. Homeopathic and conventional treatment for acute respiratory and ear complaints: a comparative study on outcome in the primary care setting. BMC Complement Altern Med 2007; 7: 7

[17] Thinesse-Mallwitz M, Maydannik V, Keller $T$ et al. A Homeopathic Combination Preparation in the Treatment of Feverish Upper Respiratory Tract Infections: An International Randomized Controlled Trial. Forsch Komplementmed 2015; 22: 163-170

[18] Grimaldi-Bensouda L, Bégaud B, Rossignol $M$ et al. Management of upper respiratory tract infections by different medical practices, including homeopathy, and 
consumption of antibiotics in primary care: the EPI3 cohort study in France 2007-2008. PLoS One 2014; 9: e89990

[19] Bellavite P, Marzotto M, Andreoli B. Homeopathic Treatments of Upper Respiratory and Otorhinolaryngologic Infections: A Review of Randomized and Observational Studies. J Altern Complement Integr Med 2019; 5: 068

[20] Weiermayer P, Frass M, Peinbauer T et al. Evidenzbasierte Veterinär-/Homöopathie und ihre mögliche Bedeutung für die Bekämpfung der Antibiotikaresistenzproblematik - ein Überblick. SAT/ASMV 2020; 162: 597-615

[21] Michael J, Singh S, Sadhukhan S et al. Efficacy of individualized homeopathic treatment of insomnia: Double-blind, randomized, placebo-controlled clinical trial. Complement Ther Med 2019; 43: 53-59

[22] Frass M, Lechleitner P, Gründling C et al. Homeopathic Treatment as an Add-On Therapy May Improve Quality of Life and Prolong Survival in Patients with Non-Small Cell Lung Cancer: A Prospective, Randomized, Placebo-Controlled, Double-Blind, Three-Arm, Multicenter Study. Oncologist 2020; 25: e1930-e55

[23] Bagot JL, Delègue C. My best case: homeopathic management of adverse effects of tamoxifen. Wien Med Wochenschr 2020; 170: 224-229

[24] Frass M, Friehs H, Thallinger C et al. Influence of adjunctive classical homeopathy on global health status and subjective wellbeing in cancer patients - A pragmatic randomized controlled trial. Complement Ther Med 2015; 23: 309-317

[25] Menut V, Seigneur E, Gras Leguen C et al. [Complementary and alternative medicine use in two French pediatric oncology centers: A common practice]. Bull Cancer 2019; 106: 189-200

[26] Samuels N, Freed Y, Weitzen R et al. Feasibility of Homeopathic Treatment for Symptom Reduction in an Integrative Oncology Service. Integr Cancer Ther 2018; 17: 486-492

[27] Rossi E, Di Stefano M, Firenzuoli F et al. Add-On Complementary Medicine in Cancer Care: Evidence in Literature and Experiences of Integration. Medicines (Basel) 2017; 4: 5

[28] Gaertner K, Lüer SC, Frei-Erb M et al. Complementary individual homeopathy in paediatric cancer care: A case series from a University Hospital, Switzerland. Complement Ther Med 2018; 41: 267-270

[29] Stub T, Kristoffersen AE, Overvåg G et al. Adverse effects in homeopathy. A systematic review and meta-analysis of observational studies. Explore NY 2020; S1550-8307(20)30379-7

[30] Dantas F, Rampes H. Do homeopathic medicines provoke adverse effects? A systematic review. Br Homeopath J 2000; 89 Suppl 1: S35-38

[31] Stub T, Kristoffersen AE, Alræk T et al. Risk in homeopathy: Classification of adverse events and homeopathic aggravations--A cross sectional study among Norwegian homeopath patients. Complement Ther Med 2015; 23: 535-543

[32] Steinsbekk A, Fønnebø V, Lewith G et al. Homeopathic care for the prevention of upper respiratory tract infections in children: a pragmatic, randomised, controlled trial comparing individualised homeopathic care and waiting-list controls. Complement Ther Med 2005; 13: 231-238

[33] Teut M, Lüdtke R, Schnabel K. Homeopathic treatment of elderly patients--a prospective observational study with follow-up over a two year period. BMC Geriatr 2010; 10: 10

[34] Danno K, Duru G, Vetel JM. Management of Anxiety and Depressive Disorders in Patients $\geq 65$ Years of Age by Homeopath General Practitioners versus Conventional General Practitioners, with Overview of the EPI3-LASER Study Results. Homeopathy 2018; 107: 81-89

[35] Frei H. Homeopathic treatment of multimorbid patients: a prospective outcome study with polarity analysis. Homeopathy 2015; 104: 57-65

[36] van Haselen R, Thinesse-Mallwitz $M$, Maidannyk $V$ et al. The Effectiveness and Safety of a Homeopathic Medicinal Product in Pediatric Upper Respiratory Tract Infections With Fever: A Randomized Controlled Trial. Glob Pediatr Health 2016; 3 : $2333794 \times 16654851$

[37] Relton C, Cooper K, Viksveen P et al. Prevalence of homeopathy use by the general population worldwide: a systematic review. Homeopathy 2017; 106: 69-78

[38] Beer AM, Burlaka I, Buskin S et al. Usage and Attitudes Towards Natural Remedies and Homeopathy in General Pediatrics: A Cross-Country Overview. Glob Pediatr Health 2016; 3: $2333794 \times 15625409$

[39] Beer AM. Homöopathika als Alternative zu Synthetika? MMW Fortschr Med 2018; 160: 60-62

[40] Forsa. Meinungen zur Homöopathie. Berlin: Forsa-Umfrage F20.0082/38662; 28. Feb 2020

[41] Abels C WJ, Walendzik A. Die Abbildung der Therapie mit Naturheilmitteln in der ärztlichen Aus- und Weiterbildung sowie in der Versorgungsforschung. IBES Diskussionsbeitrag Nr 231; 2020. Im Internet: https:// www.wiwi.uni-due.de/fileadmin/fileupload/ WIWI/Forschung/IBES_Diskussionbeitraege/IBES_Diskussionsbeitrag_Nr_231.pdf, Stand: 19.01.2021

[42] Kass B, Icke K, Witt CM et al. Effectiveness and cost-effectiveness of treatment with additional enrollment to a homeopathic integrated care contract in Germany. BMC Health Serv Res 2020; 20: 872

[43] Securvita Krankenkasse. Langzeitstudie: Homöopathie ist wirtschaftlich und wirksam. 2020. Im Internet: https://www. krankenkassen-direkt.de/news/mitteilung/ SECURVITA-Langzeitstudie-Homoeopathieist-wirtschaftlich-und-wirksam-2692040. html, Stand: 14.02.2021

[44] Rossi E, Crudeli L, Endrizzi C et al. Costbenefit evaluation of homeopathic versus conventional therapy in respiratory diseases. Homeopathy 2009; 98: 2-10

\section{Bibliografie}

Gesundh ökon Qual manag 2021; 26: 245-247 DOI 10.1055/a-1587-2308

ISSN 1432-2625

(C) 2021. The Author(s). This is an open access article published by Thieme under the terms of the Creative Commons Attribution License, permitting unrestricted use, distribution, and reproduction so long as the original work is properly cited. (https://creativecommons.org/ licenses/by/4.0/) Georg Thieme Verlag KG, Rüdigerstraße 14 70469 Stuttgart, Germany

\section{(ㄷ) (1)}

